\title{
Effectiveness of fibrolysis diacutaneous in the management of musculoskeletal diseases. A systematic review
}

\author{
Aiguade $\mathrm{R}^{1,2,4}$, Labata $\mathbf{N}^{1}$, Moure $\mathrm{L}^{1}$, Calvo $\mathrm{X}^{1}$, Pampin $\mathrm{P}^{1,4}$ and Llurda-Almuzara $\mathrm{L}^{3,4 *}$ \\ ${ }^{1}$ Nursing and Physiotherapy Departament, University of Lleida, Spain \\ ${ }^{2}$ Tecnocampus Mataró, Barcelona, Spain \\ ${ }^{3}$ Department of Basics Science, International University of Catalonia. Sant Cugat del Vallès, Spain \\ ${ }^{4}$ Institute of Fisioformación, Spain
}

\begin{abstract}
Purpose: The principal aim of the review is to study the evidence regarding the effectiveness of diacutaneous Fibrolysis (DF) in musculoskeletal diseases and getting some recommendations evidences based.

Methods: An electronic literature search was conducted utilizing PubMed, ScienceDirect, PEDRO, Cochrane and Mendeley. All titles and abstracts were reviewed, and full text articles meeting eligibility criteria were assessed in detail to determine inclusion or exclusion. Articles were reviewed by two different investigators.

Results: Principal findings of this review shows an improvement in functional capacity and a significant reduction of symptoms in the FD groups, immediately after intervention and at least, three months follow up.
\end{abstract}

Conclusions: There is limited evidence and more studies are needed to assess the effectiveness of DF to improve the function or the symptomatology.

\section{Background}

Musculoskeletal diseases are one of the main causes of disability in the developed world and consume a large amount of health and social resources, calculated around 215 billion US $\$$ in US, 26 billion Canadian \$ in Canada, 38 billion euros in Germany [1], 171,1 million US\$ in Colombia [2] or 5,7 billion pounds in UK [3].

The diacutaneous fibrolysis (FD) technique is a non-invasive physiotherapeutic technique which aims to release adherences between musculoskeletal system tissues using as a tools a set of hooks. The principal aim of the technique is to recover the optimal fascial system mobility.

The clinical use of the fibrolysis diacutaneous to treat musculoskeletal diseases is growing and it is achieving really good results. However, the scientific evidence is still poor.

\section{Purpose}

The aim of this systematic review is to explore the evidence regarding the effectiveness of diacutaneous fibrolysis in musculoskeletal diseases and getting some recommendations evidence based.

\section{Methods}

An electronic bibliographic search was performed using the next databases: PubMed $(n=7)$, ScienceDirect $(n=8)$, PEDro $(n=5)$, Cochrane $(\mathrm{n}=11)$, Mendeley $(\mathrm{n}=35)$.

The keyword "diacutaneous fibrolysis" was used. The articles found in the first search were analysed following the next inclusion criteria: published in English language, human subjects, randomized controlled trials, an intervention group treated by FD, patients suffering a musculoskeletal disease.
Using the PEDro scale, two different investigators analysed the quality of methodology in articles found in our research to be accepted in our inclusion criteria.

\section{Results}

The first search yielded 66 articles but only 4 satisfied the inclusion criteria after the first reading [4-7]. The studies selected had a methodological average score of 9 in PEDro scale (scale range 0-11, best possible score was 11).

All these 4 studies included in our revision demonstrated a clear improvement in functional capacity in the FD groups immediately after intervention. Moreover, the improvement lasted three months follow-up in two studies (one of the others two didn't assess the midterm follow-up).

Two articles demonstrated significant reduction of symptoms in the FD group. The other two didn't find this symptoms release.

\section{Conclusion}

There's limited evidence showing the symptom improvement using DF immediately after the intervention compared to conventional or placebo treatment.

${ }^{\star}$ Correspondence to: Llurda-Almuzara Luis, Anatomy Unit, Department of Basics Science, International University of Catalonia, Barcelona, Spain, Tel: 695156515, E-mail: luisllurda@gmail.com

Key words: fibrolysis diacutaneous, musculoskeletal diseases, functional capacity, symptom

Received: February 28, 2019; Accepted: March 18, 2018; Published: March 26, 2018 
More studies are needed in the assessment of DF effectiveness to improve functionality or symptomatic conditions to draw firm conclusions, especially long-term reviews. Furthermore, studies should focus on specific injuries to draw more particular conclusions about the use of FD in that injury.

\section{Implications}

We recommend fibrolysis diacutaneous, compared to control or placebo, for improving functional capacity and symptom conditions in patients suffering musculoskeletal diseases.

\section{References}

1. Da Costa BR, Vieira ER (2010) Risk Factors for Work-Related Musculoskeletal Disorders: A Systematic Review of Recent Longitudinal Studies. Am J Ind Med 53: 285-323. [Crossref]

2. Piedrahita H (2006) Costs of Work-Related Musculoskeletal Disorders (MSDs) in Developing Countries: Colombia Case. Int J Occup Saf Ergon 12: 379-386. [Crossref]
3. Buckle P (2005) Ergonomics and musculoskeletal disorders: overview. Occup Med (Lond) 55: 164-167. [Crossref]

4. López-de-Celis C, Barra-López ME, González-Rueda V, Bueno-Gracia E, RodríguezRubio PR, et al. (2018) Effectiveness of diacutaneous fibrolysis for the treatment of chronic lateral epicondylalgia: a randomized clinical trial. Clin Rehabil. 32: 644-653. [Crossref]

5. Barra López ME, López de Celis C, Fernández Jentsch G, Raya de Cárdenas L, Lucha López MO, et al. (2013) Effectiveness of Diacutaneous Fibrolysis for the treatment of subacromial impingement syndrome: a randomised controlled trial. Man Ther 18: 418-424. [Crossref]

6. Barra ME, López C, Fernández G, Murillo E, Villar E, et al. (2011) The immediate effects of diacutaneous fibrolysis on pain and mobility in patients suffering from painful shoulder: a randomized placebo-controlled pilot study. Clin Rehabil 25: 339348. [Crossref]

7. Jiménez Del Barrio S, Estébanez de Miguel E, Bueno Gracia E, Haddad Garay M, Tricás Moreno JM, et al. (2018) Effects of diacutaneous fibrolysis in patients with mild to moderate symptomatic carpal tunnel syndrome: a randomized controlled trial. Clin Rehabil 32: 1645-1655. [Crossref]

Copyright: @2019 Aiguade R. This is an open-access article distributed under the terms of the Creative Commons Attribution License, which permits unrestricted use, distribution, and reproduction in any medium, provided the original author and source are credited. 